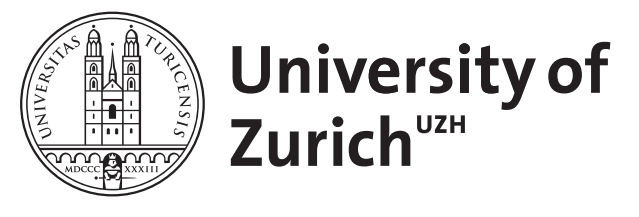

\title{
Bhabha scattering at NNLO
}

Bonciani, R ; Ferroglia, A

\begin{abstract}
We review the status of the calculation of next-to-next-to-leading order corrections to large angle Bhabha scattering in pure QED. After discussing the electron-loop and photonic corrections, we focus on the recently calculated two-loop virtual corrections involving an heavy-flavor fermion loop. We conclude by assessing the numerical impact of these corrections on the Bhabha scattering cross section at colliders operating at a center of mass energy of about $1-\mathrm{GeV}$
\end{abstract}

DOI: https://doi.org/10.1016/j.nuclphysbps.2008.09.047

Posted at the Zurich Open Repository and Archive, University of Zurich

ZORA URL: https://doi.org/10.5167/uzh-13577

Journal Article

Accepted Version

Originally published at:

Bonciani, R; Ferroglia, A (2008). Bhabha scattering at NNLO. Nuclear Physics B - Proceedings Supplements, 181:259-263.

DOI: https://doi.org/10.1016/j.nuclphysbps.2008.09.047 


\title{
Bhabha Scattering at NNLO *
}

\author{
R. Bonciania and A. Ferroglia ${ }^{a}$

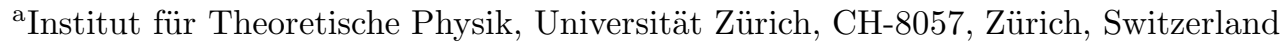

We review the status of the calculation of next-to-next-to-leading order corrections to large angle Bhabha scattering in pure QED. After discussing the electron-loop and photonic corrections, we focus on the recently calculated two-loop virtual corrections involving a heavy-flavor fermion loop. We conclude by assessing the numerical impact of these corrections on the Bhabha scattering cross section at colliders operating at a center of mass energy of about $1-\mathrm{GeV}$.

\section{INTRODUCTION}

The scattering process $e^{-} e^{+} \rightarrow e^{-} e^{+}$is named after the author that first calculated its cross section in 1936 [1]. Nowadays, Bhabha scattering is of crucial importance in the study of elementary particle phenomenology, since it is the process chosen for the luminosity determination at all $e^{+} e^{-}$colliders. In particular, at colliders operating at a center of mass energy $\sqrt{s} \sim 1-10 \mathrm{GeV}$, the luminosity is measured by observing Bhabha events at large scattering angles. In this kinematic region, the Bhabha scattering cross section is large and QED dominated: these two properties allow precise experimental measurements and an accurate theoretical evaluation of the cross section in perturbation theory. Any realistic calculation of the theoretical Bhabha scattering cross section must take into account the detector geometry and experimental cuts. For this purpose a number of sophisticated MC generators were developed $[2,3,4,5,6]$. In particular, at KLOE/DA $\Phi N E$, the experimental accuracy reached on the measurement of Bhabha scattering events, joined with the theoretical accuracy of the MC generator BABAYAGA $[3,4,7]$, allowed to determine the luminosity with an error of $0.1 \%$ [8]. Since the theoretical error on the Bhabha scattering differential cross section directly affects the luminosity measurement precision, in recent years a significant effort was devoted to

\footnotetext{
*Work supported by the SNF under contract 200020117602. Zürich preprint number ZH-TH 06/08.
}

the calculation of perturbative corrections to this process. The next-to-leading order corrections are well known even within the full Standard Model [9]. At the next-to-next-to-leading order (NNLO), for what concerns the electroweak corrections, only the logarithmically enhanced terms were calculated [10]. In pure QED, instead, the situation is sensibly different. The first diagrammatic calculation of the two-loop QED virtual corrections to Bhabha scattering can be found in [11]. However, this result was obtained by employing dimensional regularization (DR) to regulate both soft and collinear divergencies, while all the available MC generators employ the electron mass $m_{e}$ as a collinear regulator. Today, the complete set of NNLO corrections to Bhabha scattering in pure QED have been evaluated using $m_{e}$ as a collinear regulator. The two-loop Feynman diagrams involved in the calculation can be divided in three gauge independent sets: i) diagrams without fermion loops ("photonic" diagrams), ii) diagrams involving a closed electron loop, and iii) diagrams involving a closed loop of a fermion heavier than the electron. In the following three sections we consider the calculation of each set of corrections separately. In Section 5, we conclude by briefly discussing the numerical impact of these corrections on the Bhabha scattering cross section at $\sqrt{s} \sim 1 \mathrm{GeV}$. 


\section{PHOTONIC CORRECTIONS}

All the NNLO photonic corrections, with the exception of the ones arising from the two-loop photonic boxes, can be obtained in a straightforward way, retaining the full dependence on $m_{e}$ [12], by using a technique that is by now standard in multi-loop calculations. This technique is based on the Laporta algorithm [13] for the reduction of the Feynman diagrams to the Master Integrals (MIs), and then on the differential equation method [14] for their analytic evaluation. However, a calculation of the two-loop photonic boxes retaining the full dependence on $m_{e}$ is beyond the reach of this technique. This is due to the fact that the number of Master Integrals belonging to the same topology after the reduction process is large ${ }^{2}$. This means that one should be able to solve analytically large systems of first-order ordinary differential equations; this is in general not possible. Alternatively, one could use the Mellin-Barnes techniques [15] to calculate the MIs one by one $e^{3}$. The calculation, however, is very complicated and, moreover, from a phenomenological point of view, to keep the full dependence on the electron mass is not important. In fact, the physical problem exhibits a well defined mass hierarchy. The mass of the electron is always very small compared to the other kinematic invariants and it can be safely neglected everywhere, with the exception of the terms in which it acts as a collinear regulator. It can be shown that the collinear structure of the NNLO corrections is the following

$\frac{d \sigma^{(N N L O)}}{d \sigma^{(\mathrm{Born})}}=\frac{\alpha^{2}}{\pi^{2}} \sum_{i=0}^{2} \delta^{(i)} L_{e}^{i}+\mathcal{O}\left(\frac{m_{e}^{2}}{s}, \frac{m_{e}^{2}}{t}\right)$

where $L_{e}=\ln \left(s / m_{e}^{2}\right)$ and where the coefficients $\delta^{(i)}$ are functions of the scattering angle $\theta$ and, in general, of the mass of the heavy fermions involved in the virtual corrections. For any practical purpose, the approximation given by Eq. (1) is sufficient for a phenomenological description of the process ${ }^{4}$. In the photonic case, the coeffi-

\footnotetext{
${ }^{2}$ Some topologies have up to 6 MIs.

${ }^{3}$ Partial results can be found in $[16,17]$

${ }^{4}$ It can be shown that the terms suppressed by a positive power of $m_{e}^{2} / s$ do not play any phenomenological role
}

cients of the squared and single collinear logarithm in Eq. (1) were obtained in $[18,19]$. The precision required for luminosity measurements at $e^{+} e^{-}$colliders demanded the calculation of the non-logarithmic coefficient, that was obtained in [20] through the infrared matching to the massless approximation. The technique of [20] allowed to reconstruct the photonic differential cross section in the $s \gg m_{e}^{2} \neq 0$ limit from the calculation in [11], where $m_{e}$ was set to zero from the start. The method employed in [20] involves a change of regularization scheme for the collinear divergencies originating from a vanishing electron mass. A method based on a similar principle was subsequently developed in [21,22]; the authors of [22] confirmed the result of [20] for the NNLO photonic corrections to the Bhabha scattering differential cross section.

\section{ELECTRON LOOP CORRECTIONS}

The NNLO electron loop corrections arise from the interference of two-loop Feynman diagrams with the tree-level amplitude as well as from the interference of one-loop diagrams, as long as one of the diagrams contributing to each term involves a closed electron loop. This set of corrections presents a single two-loop box topology, and it is therefore technically less challenging to evaluate with respect to the photonic correction set. The calculation of the electron loop corrections was completed a few years ago [23]; the final result retains the full dependence of the differential cross section on the electron mass $m_{e}$. The Master Integrals involved in the calculation were identified by means of the Laporta algorithm and evaluated with the differential equation method. As expected, after UV renormalization the differential cross section presented only residual IR poles that were removed by adding the contribution of the soft photon emission diagrams. The resulting NNLO differential cross section could be conveniently written in terms of 1 - and 2dimensional Harmonic Polylogarithms (HPLs) of

already at very low c.m. energies, $\sqrt{s} \sim 10 \mathrm{MeV}$. Moreover, the terms $m_{e}^{2} / t$ (or $m_{e}^{2} / u$ ) become important in the very forward (backward) region, unreachable for the experimental set ups. 
maximum weight three [24]. Expanding the cross section in the limit $s,|t| \gg m_{e}^{2}$, the ratio of the NNLO corrections to the Born cross section can be written as in Eq. (1):

$\frac{d \sigma^{(2, \mathrm{EL})}}{d \sigma^{(\mathrm{Born})}}=\frac{\alpha^{2}}{\pi^{2}} \sum_{i=0}^{3} \delta^{(\mathrm{EL}, \mathrm{i})} L_{e}^{i}+\mathcal{O}\left(\frac{m_{e}^{2}}{s}, \frac{m_{e}^{2}}{t}\right)$.

The explicit expression of all the coefficients $\delta^{(\mathrm{EL}, \mathrm{i})}$, obtained by expanding the results of [23] was confirmed by two different groups $[22,25]$. It is easy to check that the cubed collinear logarithm in Eq. (2) cancels against the corresponding term arising from the soft pair production graphs [26].

\section{HEAVY FLAVOR CORRECTIONS}

Finally, we consider the corrections originating from two-loop Feynman diagrams involving a heavy flavor fermion loop ${ }^{5}$. Since this set of corrections involves one more mass scale with respect to the corrections analyzed in the previous sections, a direct diagrammatic calculation is in principle a more challenging task. Recently, Becher and Melnikov applied their technique based on SCET to Bhabha scattering and obtained the heavy flavor NNLO corrections in the limit in which $s,|t|,|u| \gg m_{f}^{2} \gg m_{e}^{2}$, where $m_{f}^{2}$ is the mass of the heavy fermion running in the loop [22]. Their result was very soon confirmed by means of a method based on the asymptotic expansion of Mellin Barnes representation of the Master Integrals involved in the calculation [25]. However, the results obtained in the approximation $s,|t|,|u| \gg m_{f}^{2} \gg m_{e}^{2}$ cannot be applied to the case in which the $\sqrt{s}<m_{f}$ (as in the case of a tau loop at $\sqrt{s} \sim 1 \mathrm{GeV}$ ), and they apply only to a relatively narrow angular region perpendicular to the beam direction when $\sqrt{s}$ is not very much larger than $m_{f}$ (as in the case of top quark loops at ILC). It was therefore necessary to calculate the heavy flavor corrections to Bhabha scattering assuming only that $s,|t|,|u|, m_{f}^{2} \gg m_{e}^{2}$. The technical problem can be simplified by considering carefully, once more, the structure of the

\footnotetext{
${ }^{5}$ In this context, and at $\sqrt{s} \sim 1 \mathrm{GeV}$, by heavy flavor fermion we mean muon and tau leptons, as well as $b$ - and $c$-quarks. Top quarks completely decouple at intermediate energies.
}

collinear singularities of this set of corrections. The ratio of the NNLO heavy flavor corrections to the Born cross section is given by

$\frac{d \sigma^{(2, \mathrm{HF})}}{d \sigma^{(\text {Born })}}=\frac{\alpha^{2}}{\pi^{2}} \sum_{i=0}^{1} \delta^{(\mathrm{HF}, \mathrm{i})} L_{e}^{i}+\mathcal{O}\left(\frac{m_{e}^{2}}{s}, \frac{m_{e}^{2}}{t}\right)$.

It is possible to prove that, in a physical gauge, all the collinear singularities factorize and can be absorbed in the external field renormalization [27]. This observation has two consequences in the case at hand. The first one is that box diagrams are free of collinear divergencies in a physical gauge; since the sum of all boxes forms a gauge independent block, it can be concluded that the sum of all box diagrams is free of collinear divergencies in any gauge. The second consequence is that the single collinear logarithm in Eq. (3) arises from vertex corrections only. Moreover, if one chooses on-shell UV renormalization conditions, the irreducible two loop vertex graphs are free of collinear singularities. Therefore, among all the two-loop diagrams contributing to the NNLO heavy flavor corrections to Bhabha scattering, only the reducible vertex corrections are logarithmically divergent in the $m_{e} \rightarrow 0$ limit $^{6}$, and they are very easy to calculate even if they depend on two different masses. By exploiting these two facts, we were recently able to obtain the NNLO heavy flavor corrections to the Bhabha scattering differential cross section $[28,29]$, assuming only that $s,|t|,|u|, m_{f}^{2} \gg m_{e}^{2}$. In particular, in obtaining the analytic expression for the NNLO cross section, we worked in the Feynman gauge, setting $m_{e}=0$ from the start in all the diagrams with the exception of the reducible ones and of the interference of one-loop graphs. This procedure allowed us to effectively eliminate a mass scale from the two-loop boxes, so that the evaluation could be carried out with the techniques already employed in the diagrammatic calculation of the electron loop corrections ${ }^{7}$. We want to stress that in this approach individual box diagrams are singular in the $m_{e} \rightarrow 0$ limit and the collinear singularities appear as additional poles in the dimen-

\footnotetext{
${ }^{6}$ Additional collinear logarithms arise also from the interference of one-loop vertex and self-energy diagrams.

${ }^{7}$ The necessary MIs can be found in $[29,30]$
} 
sional regulator $\epsilon$; however it is easy to prove that such divergencies cancel in the sum of all the box diagrams. By expanding the analytic results of $[28,29]$ it was possible to check the heavy flavor cross section in the $s,|t|,|u| \gg m_{f}^{2} \gg m_{e}^{2}$ limit, which was previously known $[22,25]$. At intermediate energy colliders like DA $\Phi \mathrm{NE}$, the exact dependence on $m_{f}$ of the results of $[28,29]$ allows to account for the contribution of muons, taus, $b$ and $c$-quark loops to the Bhabha scattering cross section. In the case in which the heavy flavor fermion is a quark, it was straightforward to modify the calculation of the two-loop self-energy diagrams to obtain the mixed QED-QCD corrections to Bhabha scattering [29]. An alternative numerical approach to the calculation of the heavy flavor corrections to Bhabha scattering, based on dispersion relations, was pursued in [31]. The latter method allows also to evaluate the contribution of the light quarks vacuum polarization to the Bhabha scattering cross section; to this purpose one has to convolute the kernel functions with the data concerning the cross section of the process $e^{+} e^{-} \rightarrow$ hadrons.

\section{CONCLUSIONS}

The numerical impact of the photonic and electron loop QED corrections to the Bhabha scattering cross section at flavor factories was carefully examined in $[4,7]$. The authors were able to show that the event generator BABAYAGA, based of the matching of exact NLO corrections with the Parton Shower algorithm, has a theoretical accuracy of the order of $0.1 \%$. A similar analysis of the heavy flavor NNLO corrections is not yet available. However, it is possible to evaluate numerically the NNLO heavy flavor corrections to the Bhabha scattering cross section. In Table 1 we show the results of such an evaluation for $\sqrt{s}=1$ $\mathrm{GeV}$ and for a scattering angle $\theta$ in the range $50^{\circ}<\theta<130^{\circ}$ (see [29] for details). It is possible to observe that the muon corrections are an order of magnitude larger of the corrections involving heavier fermions while they are one order of magnitude smaller than the electron loop corrections and they reach $1 / 2$ permille of the Born cross section at large scattering angles.
In conclusion, the calculation of the two-loop corrections to Bhabha scattering in QED is now complete. The calculation of the heavy fermion NNLO corrections allowed to remove the last piece of pure theoretical uncertainty in the luminosity determination at low-energy accelerators.

\section{REFERENCES}

1. H. J. Bhabha, Proc. Roy. Soc. Lond. A $\mathbf{1 5 4}$ (1936) 195.

2. F. A. Berends and R. Kleiss, Nucl. Phys. B 228 (1983) 537. F. A. Berends, R. Kleiss and W. Hollik, Nucl. Phys. B 304 (1988) 712.

3. C. M. Carloni Calame, C. Lunardini, G. Montagna, O. Nicrosini and F. Piccinini, Nucl. Phys. B 584 (2000) 459. C. M. Carloni Calame, Phys. Lett. B 520, (2001) 16. C. M. Carloni Calame, G. Montagna, O. Nicrosini and F. Piccinini, Nucl. Phys. Proc. Suppl. 131 (2004) 48.

4. G. Balossini, C. M. Carloni Calame, G. Montagna, O. Nicrosini and F. Piccinini, Nucl. Phys. B 758 (2006) 227.

5. S. Jadach, W. Placzek and B. F. L. Ward, Phys. Lett. B 390 (1997) 298. W. Placzek, S. Jadach, M. Melles, B. F. L. Ward and S. A. Yost, arXiv:hep-ph/9903381.

6. A. B. Arbuzov, G. V. Fedotovich, E. A. Kuraev, N. P. Merenkov, V. D. Rushai and L. Trentadue, JHEP 9710 (1997) 001.

7. C. M. Carloni Calame, these proceedings.

8. A. Aloisio et al. [KLOE Collaboration], Phys. Lett. B 606 (2005) 12.

9. M. Consoli, Nucl. Phys. B 160 (1979) 208. M. Bohm, A. Denner and W. Hollik, Nucl. Phys. B 304 (1988) 687. F. A. Berends and R. Kleiss, Nucl. Phys. B 228 (1983) 537. M. Caffo, R. Gatto and E. Remiddi, Nucl. Phys. B 252 (1985) 378.

10. J. H. Kuhn, A. A. Penin and V. A. Smirnov, Eur. Phys. J. C 17 (2000) 97. Nucl. Phys. Proc. Suppl. 89 (2000) 94. J. H. Kuhn, S. Moch, A. A. Penin and V. A. Smirnov, Nucl. Phys. B 616 (2001) 286 [Erratum-ibid. B 648 (2003) 455]. B. Feucht, J. H. Kuhn, A. A. Penin and V. A. Smirnov, Phys. Rev. Lett. 93 (2004) 101802. B. Jantzen, 


\begin{tabular}{|c|c|c|c|c|c|c|}
\hline$\theta$ & phot $\left(10^{-4}\right)$ & $e\left(10^{-4}\right)$ & $\mu\left(10^{-4}\right)$ & $c\left(10^{-4}\right)$ & $\tau\left(10^{-4}\right)$ & $b\left(10^{-4}\right)$ \\
\hline $50^{\circ}$ & 36.688225 & 17.341004 & 1.7972877 & 0.0622677 & 0.0264013 & 0.0010328 \\
\hline $70^{\circ}$ & 41.240039 & 19.438718 & 2.6504950 & 0.1086126 & 0.0465329 & 0.0018907 \\
\hline $90^{\circ}$ & 45.780639 & 21.463240 & 3.4581845 & 0.1321857 & 0.0576348 & 0.0024428 \\
\hline $110^{\circ}$ & 49.366078 & 23.099679 & 4.0922189 & 0.1098317 & 0.0495028 & 0.0022024 \\
\hline $130^{\circ}$ & 50.349342 & 23.847394 & 4.4392717 & 0.0549436 & 0.0273145 & 0.0013297 \\
\hline
\end{tabular}

Table 1

The second-order electron, muon, $c$-quark, $\tau$-lepton, and $b$-quark QED contributions to the differential cross section of Bhabha scattering at $\sqrt{s}=1 \mathrm{GeV}$ in units of $10^{-4}$ of the Born cross section.

J. H. Kuhn, A. A. Penin and V. A. Smirnov, Phys. Rev. D 72 (2005) 051301 [Erratumibid. D 74 (2006) 019901]. Nucl. Phys. B 731 (2005) 188 [Erratum-ibid. B 752 (2006) 327].

11. Z. Bern, L. Dixon, and A. Ghinculov, Phys. Rev. D 63 (2001) 053007.

12. R. Bonciani and A. Ferroglia, Phys. Rev. D 72 (2005) 056004.

13. S. Laporta and E. Remiddi, Phys. Lett. B 379 (1996) 283. S. Laporta, Int. J. Mod. Phys. A 15 (2000) 5087. F. V. Tkachov, Phys. Lett. B 100 (1981) 65. G. Chetyrkin and F. V. Tkachov, Nucl. Phys. B 192 (1981) 159.

14. A. V. Kotikov, Phys. Lett. B 254 (1991) 158; Phys. Lett. B 259 (1991) 314; Phys. Lett. B 267 (1991) 123. E. Remiddi, Nuovo Cim. A 110 (1997) 1435. M. Caffo, H. Czyz, S. Laporta and E. Remiddi, Acta Phys. Polon. B 29 (1998) 2627. Nuovo Cim. A 111 (1998) 365. M. Argeri and P. Mastrolia, Int. J. Mod. Phys. A 22 (2007) 4375.

15. V. A. Smirnov, Evaluating Feynman Integrals (Springer, Berlin, 2004). S. Friot, et al., Phys. Lett. B 628 (2005) 73.

16. V. A. Smirnov, Phys. Lett. B 524, 129 (2002). G. Heinrich and V. A. Smirnov, Phys. Lett. B 598, 55 (2004). M. Czakon, J. Gluza and T. Riemann, Phys. Rev. D 71, 073009 (2005). Nucl. Phys. B 751, 1 (2006).

17. D. J. Broadhurst, J. Fleischer and O. V. Tarasov, Z. Phys. C 60 (1993) 287. A. I. Davydychev and M. Y. Kalmykov, Nucl. Phys. B 699 (2004) 3.

18. A. B. Arbuzov, E. A. Kuraev and B. G. Shaikhatdenov, Mod. Phys. Lett. A 13 (1998) 2305.
19. E. W. N. Glover, J. B. Tausk and J. J. Van der Bij, Phys. Lett. B 516 (2001) 33.

20. A. A. Penin, Phys. Rev. Lett. 95 (2005) 010408. Nucl. Phys. B 734 (2006) 185.

21. A. Mitov, S. Moch, JHEP 0705 (2007) 001.

22. T. Becher and K. Melnikov, JHEP 0706 (2007) 084.

23. R. Bonciani, P. Mastrolia and E. Remiddi, Nucl. Phys. B 661 (2003) 289 [Erratum-ibid. B 702 (2004) 359]. Nucl. Phys. B 690 (2004) 138. Nucl. Phys. B 676 (2004) 399. R. Bonciani, A. Ferroglia, P. Mastrolia, E. Remiddi and J. J. van der Bij, Nucl. Phys. B 681 (2004) 261 [Erratum-ibid. B 702 (2004) 364]. Nucl. Phys. B 701 (2004) 121. Nucl. Phys. B 716 (2005) 280.

24. A .B. Goncharov, Math. Res. Lett. 5 (1998), 497-516. D. J. Broadhurst, Eur. Phys. J. C 8 (1999) 311. E. Remiddi and J. A. M. Vermaseren, Int. J. Mod. Phys. A 15 (2000) 725. T. Gehrmann and E. Remiddi, Comput. Phys. Commun. 141 (2001) 296. Comput. Phys. Commun. 144 (2002) 200. D. Maître, Comput. Phys. Commun. 174 (2006) 222. arXiv:hep-ph/0703052.

25. S. Actis, M. Czakon, J. Gluza and T. Riemann, Nucl. Phys. B 786 (2007) 26.

26. A. B. Arbuzov, E. A. Kuraev, N. P. Merenkov and L. Trentadue, Phys. Atom. Nucl. 60 (1997) 591 [Yad. Fiz. 60N4 (1997) 673].

27. J. Frenkel and J. C. Taylor, Nucl. Phys. B 116 (1976) 185.

28. R. Bonciani, A. Ferroglia and A. A. Penin, Phys. Rev. Lett. 100 (2008) 131601.

29. R. Bonciani, A. Ferroglia and A. A. Penin, JHEP 0802, 080 (2008). 
30. J. Fleischer, A. V. Kotikov and O. L. Veretin, Nucl. Phys. B 547 (1999) 343. U. Aglietti and R. Bonciani, Nucl. Phys. B 668 (2003) 3. Nucl. Phys. B 698 (2004) 277.

31. S. Actis, M. Czakon, J. Gluza and T. Riemann, Phys. Rev. Lett. 100 (2008) 131602. 